\title{
Evaluation of Quality of Neonatal Intensive Care Unit Service in Primary Hospitals of Jimma Zone, Southwest Ethiopia
}

\section{Lamesa Fikadu}

CORDAID International

Kidist Adamu

Jimma University

Yosef Haile ( $\sim$ yosefjh2017@yahoo.com )

Arba Minch University

\section{Research Article}

Keywords: Evaluation, Quality, Neonatal intensive care unit, Jimma zone, primary hospital

Posted Date: February 24th, 2022

DOI: https://doi.org/10.21203/rs.3.rs-1373561/v1

License: (c) (i) This work is licensed under a Creative Commons Attribution 4.0 International License.

Read Full License 


\section{Abstract}

Background: Neonatal Intensive Care Unit (NICU) is a health facility based package of interventions to address newborns that need advanced care for ill or premature new-borns in the first 28 days of life. The period with in the first month of life was the most important time for child's survival and they face the highest risk of death. There is paucity of information regarding this important issue in south west Ethiopia therefore, it is very important to evaluate quality of neonatal Intensive Care Unit services especially in the era of mid covid-19 pandemic. Hence, the aim of this study was to evaluate the quality of neonatal intensive care unit service at primary hospitals in Jimma zone, 2020.

Methods: A single case study using both quantitative and qualitative data collection methods was conducted from August 20 to September 15, 2020 in neonatal intensive care units of primary hospitals located in Jimma zone. The evaluation was done using Donabedian's Structure-Process-Outcome model in terms of availability, compliance and acceptability dimensions. Data were entered in EpiData version 3.1 and analyzed using SPSS version 25. Descriptive statistics was used to summarize the data. Bivariate and multivariable logistic regression analyses were performed to see the association between independent variable and satisfaction of parents on quality of service. The quantitative findings were supplemented with qualitative data. Finally the findings of the evaluation were interpreted on the basis of pre-determined judgment matrix.

Results: Most of the required resources (88.32\%) were available at the study sites. But the rooms had been inadequate. Health care providers' compliance was $82.59 \%$. However, after discharge, only $26.67 \%$ of neonates received appointment based on guideline. About $72.75 \%$ of parents were satisfied with the services given to their babies. The overall quality of neonatal intensive care service was partially implemented (82.42\%) according to the preset judgment criteria. Parental satisfaction was found to be associated with place of residence (AOR=3.68, $95 \% \mathrm{Cl}$ : 1.15-11.76) and occupation (AOR=2.69, 95\% Cl: 1.01-7.2).

Conclusions and Recommendations: The overall quality of neonatal intensive care service was partially implemented according to the preset judgment criteria. Rural residence and having no occupation were predictors for parenteral satisfaction. There is a need for improvements on preparation of maternal waiting area, hand washing facilities, provision of appointments to discharged neonates and the availability of medical equipment's (Continuous Positive Airway Pressure).

\section{Introduction}

Neonatal mortality, accounting for an estimated 2.5 million deaths worldwide in 2018 and an estimated 18 neonatal death per 1,000 live births were occurred and it takes the largest share of under-five mortality and accounts for $47 \%$. The highest neonatal mortality occurred in Sub-Saharan Africa and Southern Asia which accounts 28 and 25 deaths per 1000 live births, respectively. A child born in sub-Saharan Africa is 10 times more likely to die in the first month of life than a child born in high-income country (1). 
The leading causes of neonatal deaths were congenital anomalies and prematurity in higher-income countries whereas preterm birth, intra partum-related complications (lack of breathing at birth), infections and birth defects caused most neonatal deaths in 2018 in middle and low income countries (1). Two third of the newborn deaths in Africa would be preventable through enhanced implementation of maternal and child health services (2).

In Ethiopia, under-five mortality rate has declined from 67 to 55 per 1,000 live births between 2016 and 2019. Similarly infant mortality rate has also decreased from 48/1,000 live births in 2016 to $43 / 1,000$ live births in 2019. However, neonatal mortality rate shows increment from 29 to 30 per 1,000 live births between similar years (3). Nearly $44 \%$ of under-five deaths in Ethiopia occurred within 28 days of life which contains larger proportion (4). The three common causes of neonatal deaths in Ethiopia are birth prematurity, sepsis and asphyxia and to prevent this skilled care during childbirth, and the postpartum period are important interventions in reducing neonatal morbidity and mortality $(3,4)$.

In the study done in Jimma University Medical Center the three top causes of NICU admission were low birth weight followed by neonatal sepsis and hypothermia and the three top causes of neonatal mortality were low birth weight followed by prematurity and respiratory distress syndrome (RDS). In this study from 3093 neonates admitted in NICU during the study period 2681 (86.7\%) were recovered and 412 (13.3\%) were died. Parental residency, the length of stay, RDS, perinatal asphyxia, low birth weight, prematurity, and congenital malformations were factors associated with neonatal mortality (5). Another study conducted in Wolaita Sodo University Teaching and Referral Hospital reported that the most common causes of admission were neonatal sepsis followed by respiratory distress. From 964 neonates admitted in the NICU of the hospital 159 (16.5\%) deaths of the neonates occurred. Respiratory distress syndrome $31(66 \%)$, perinatal asphyxia $58(22 \%)$ and congenital malformation $2(5.3 \%)$ were the leading causes of death during admission (6).

Nationally, different activities have been implemented to reduce neonatal mortality including the provision of essential neonatal care (CBNC) at the community level through Health Extension Workers, establishing newborn intensive corners in primary health facilities and establishing of the neonatal intensive care unit (NICU) in hospitals (7). Ethiopia also implemented Integrated Management of Newborn and Childhood Illness (IMNCI) with the aim of to reduce mortality, illness and to promote improved growth and development among under five children. It includes both preventive and curative elements that are implemented by families and communities as well as by health facilities. It has 3 main strategies including improving case management skills of health care staff, improving the health systems and improving family and community health practices (8).

Neonatal Intensive Care Unit (NICU) is a health facility based package of interventions to address newborns that need advanced care for ill or premature new-borns in the first 28 days of life (9). The period with in the first month of life was the most important time for child's survival and they face the highest risk of death 9). Intensive Care Unit (ICU) is a specially staffed and equipped based on separate area of a hospital devoted to provide the management and monitoring of patients with life-threatening 
conditions (10). Its emergence was recognized as a means of delivering the specialized care to newborns that are in need in terms of treatment and prevention (12).

Most newborn deaths can be prevented through improved identification of those at high risk and timely provision of quality supportive care and through existing, proven, cost effective interventions: antibiotics, cord care, resuscitation, immediate and exclusive breastfeeding and keeping the newborn warm through putting the baby to the mother skin or kangaroo mother care $(K M C)(11,12)$. Provision of optimal supportive care in a hospital with Neonatal Intensive Care Units (NICUs) could avert $90 \%$ of neonatal deaths due to preterm birth complications (11)

A child risk of death is greater in the first four weeks of life than at any other time during the first 12 months of life. Therefore, neonatal intensive care unit services are very crucial (7). In a study done in Ethiopia on pre ( 6 month before the NICU intervention) and post ( 6 month after the NICU intervention) in four hospitals (Assela Hospital, Adama Hospital medical college, Nigest Eleni Mohammed memorial general hospital in Hosaena and Hawassa University college) revealed a significant improvement in neonatal health outcome after the intervention of NICU. In these studies a significant increase of $108 \%$ in NICU service utilization was observed in all the sites, the number of neonates referred to other hospitals decreased by $50 \%$, length of stay in hospital reduced from 8.3 days to 7.2 days, neonatal mortality were reduced from $8.2-6.2 \%$ and neonates with improved health status were increased from $85.3-88.1 \%$ after the intervention of NICU across the four sites (13).

However, the provision of inpatient care for sick and premature newborns need specialized care but it presents critical health system challenges (11). The major challenges in the provision of neonatal service (NICU) include insufficient funds, lack of sufficient rooms for service provision, absence of standard implementation strategies, lack of strong supportive supervision mechanisms, lack of strong referral and linkage between health facilities to provide quality services for newborn (9). The other challenges related to neonatal intensive care service provision were shortage of medical supplies, equipment and shortage of skilled health care providers (12).

To our knowledge, there is no published study available on the evaluation of quality of NICU services provided by public hospitals in South West Ethiopia. In addition, since COVID-19 pandemic has affected the provision of quality health services especially in low income countries therefore understanding the level of quality of NICU services at this time is very crucial to improve the quality of health services in the Neonatal Intensive Care Unit. Hence, this study was aimed to evaluate the quality of neonatal intensive care unit services in primary hospitals in south west Ethiopia.

\section{Evaluation Methods}

\section{Evaluation design, study area and period}

A single case study using both quantitative and qualitative data collection methods were used. The evaluation was conducted in primary hospitals located in Jimma Zone, Oromia region. The Zone is 
bounded on the south by the southern Nations, Nationalities and Peoples Region, the northwest by illubabor, on the north by East Wolega, and on the northeast by West Shewa. The majority of the population is rural while $11.31 \%$ are urban inhabitants. According to Jimma zone health office, the zone has 21 districts, 708,288 households, 120 health centres, 507 health posts, 2 general hospitals (Agaro and Limuu Genet) and 4 primary hospitals (Omo Nada, Seka Chekorsa, Sentema and Dedo hospital). It has a total population of $3,399,784$ of which $51 \%$ are women and $49 \%$. The evaluation period was from Juanary 28,2019 to July 28,2020 GC (14).

\section{Focus of the Evaluation}

The evaluation approach for this study was formative evaluation. The focus of this evaluation was on the process of program activity, on the input of the program, activities conducted and the immediate result of the activities.

\section{Population}

\section{Source population}

All primary hospitals found in Jimma zone, all parents of the neonates who came to the NICU, all health care providers who had working in NICU in Jimma zone primary hospitals, all head of the hospitals in Jimma zone with NICU service and all program documents since June, 2019 in NICU were source population.

\section{Study population}

All Primary hospitals in Jimma zone with NICU service, selected parents of the neonates who had received the NICU service during data collection period, all health care providers who had working in NICU during data collection period, CEO of primary hospitals with NICU services, child health program coordinator at Jimma zone health office and NICU registration book and 6 month reports since Juanary 28, 2019 to July 28,2020 GC were study population.

\section{Inclusion and Exclusion criteria}

Jimma zone government primary hospitals with NICU services, health care providers who had a working experience for at least 6 months in NICU in on the study site, parents of admitted neonate in NICU in Jimma zone primary hospitals during data collection period, CEO of the primary hospitals in the study site and program coordinators at zonal health office, all NICU service related documents use of between January 28,2019 to July 28, 2020 GC were included. Parents who were critically ill during data collection and unable to speak and hear parents who visit the hospital for the second time and above during data collection period were excluded from the study.

\section{Evaluation Dimensions}

In this study the structure was assessed with the availability dimension, process by compliance dimension and the outcome of the service with Acceptability dimension. The dimension of availability 
was used to assess availability of HCP, infrastructure and technical resources including medical equipment and drugs that are important for implementation of NICU program. The dimension of Compliance was used to assess content quality of program performance with pre-set standard of design or national guideline. It was assessed by direct observation and program document and record review. In this evaluation, compliance meant obedience of health facility and healthcare providers with Ethiopian guidelines in delivering NICU services.

According to Avedis Donabedian 2003, acceptability was defined as "conformity to the wishes, desires and expectations of patients and responsible members of their families" (15).

\section{Indicators}

Indicators were developed through consideration of WHO Service Availability and Readiness Assessment (SARA) 2013, Neonatal Intensive Care Unit (NICU) Training Participants' Manual 2014, St. Paul's Hospital Millennium Medical College (SPHMMC) NICU guiding protocol and adapted from a literature done in Debre birhan hospital $(16,17,18,19)$. A total of 30 indicators were used having 11 indicators for availability, 9 indicators for compliance and 10 indicators for Parental satisfaction. Prioritization of indicators were done based on nominal group technique and each of the indicators were weighted after asking a group of experts to compare each prioritized indicators against others, in terms of their relative importance for the specified dimensions.

\section{Availability Indicators are:}

Number of hospitals with at least two trained health care provider in NICU, Number of hospital's with neonatal bed in the NICU ward, Number of hospitals with NICU rooms (KMC room, intensive care room, isolation room, procedure room, waiting area, nursing station), Number of hospitals with medical equipment's needed to provide NICU service (Radiant warmer, Pulse oximetry, Suction machine, Ambubag, Thermometer, baby weight scale, stethoscope), Number of hospitals with drug supplies (Ampicillin injection, Gentamycin injection, ceftriaxone injection), Number of hospitals with functional laboratory service, Number of hospitals with NICU guideline placed in NICU room, Number of hospitals with standard NICU registration book, Number of hospitals with standard monthly reporting format, Number of hospitals with tap water in the NICU ward, and Number of hospitals with functional electricity in NICU

\section{Compliance indictors are:}

Proportion of neonates who are putted on sterile sheet on admission, Proportion of neonates who are weighted on admission, Proportion of neonates who received temperature record on admission, proportion of neonates who are continuously monitored during their admission, Proportion of neonates who are appointed within 7 days of after discharge, Proportion of parents who received counselling during admission of their neonate, Proportion of hospitals which received integrated supportive 
supervision quarterly for the last six months, Number of timely report compiled for the last 2 quarter, Number of complete report compiled for the last 2 quarter

\section{Acceptability Indicators are:}

Proportion of parents who satisfied with cleanliness of NICU, Proportion of Parents who satisfied with the availability of NICU service provider on working time, Proportion of Parents who satisfied with the staffs response about any information, proportion of Parents who satisfied with availability of neonatal bed in NICU, Proportion of Parents who satisfied with their baby's progress, Proportion of Parents who satisfied with availability of all ordered investigation, Proportion of Parents who satisfied with availability of all prescribed drug, Proportion of Parents who satisfied with supports of the staff, Proportion of Parents satisfied with room's ventilation and Proportion of Parents satisfied with adequate treatment for neonate.

\section{Sample size determination and procedure Sample size determination}

For Parental exit interview the sample size were calculated by using single population proportion formula.

$$
\begin{gathered}
\mathrm{n}=\underline{\mathrm{Z}^{2} * \mathrm{P}(1-\mathrm{P})} \\
\mathrm{d}^{2}
\end{gathered}
$$

Where:

n: Sample size

P: proportion of parents satisfied with the overall service given to their admitted neonate at the hospital. Since there was a study done on Debre Birhan Hospital on the quality of NICU service the overall satisfaction of mothers was 0.77 or $77 \%$. So, $p=77 \%$ was taken to calculate the parental satisfaction sample size.

d: margin of error $(5 \%)$

Z: Standard normal variable at 95\% confidence level (1.96)

$$
\underline{1.96^{2} * 0.77(1-0.77)}=\underline{\mathbf{2 7 2}}
$$

Sample size $(\mathrm{n})$ calculated was $=$

The three consecutive month's data on NICU users from the 3 primary hospitals indicated that, in average 170 neonates used NICU services. Since the source population is less than 10,000 we have adjusted our 
sample size using a population correction formula (16). We finally determined a total of 109 by considering $5 \%$ non-response rate.

$\mathrm{N}_{\text {final }}=\mathrm{n} /(1+(\mathrm{n} /$ population $))$

$=272$

$(1+272 / 170)$

$N_{\text {final }}=104$, by assuming $5 \%$ non-response rate the final sample size were 109 parents were interviewed.

For document and record review:

- Six months of NICU registers and reports from January 28, 2019 to July 28, 2020 GC were reviewed.

- 5 Patient vital sign sheet and discharge sheet per each hospital of the admitted neonate during data collection period was observed.

For key informant interview:

- 1 NICU program coordinator at zonal health office, 3 CEO (1 per each) of the hospital and 3 health care providers who have work experience of at least 6 month and above in NICU were included.

For observation:

- For provider and patient interaction observation: 15 sessions or patient examination (5 per each hospital) sessions were observed to check quality or compliance of patients examination session.

Resource inventory:

- Resource inventory or audit of health facilities were conducted in all (3) sampled primary hospitals in Jimma zone. It includes inventory of infrastructure, human resource, guidelines, essential drug supplies and medical equipment's, laboratory test, recording and reporting materials.

\section{Sampling Procedure}

Among the four primary hospitals located in Jimma Zone three started to provide NICU service. So, all the three primary hospitals in Jimma zone with neonatal intensive care unit service were included in the study. For the assessment of parent's satisfaction, the total sample was allocated proportionally to each 3 of the primary hospitals by considering their case flow. So, Sample size for Seka Ceqorsa primary Hospital were 42, for Sexema were 28 and for Omo Nada were 39 parents for the exit interview. The parents were interviewed consecutively at exit until the required sample was obtained. For key informant interview health care providers, heads of hospitals and program manager at zonal health departement was used to obtain information regarding program management and barriers to service implementation and solutions. Three head of hospitals (CEO) and one program coordinator at zonal health office were participated in the study. For health care providers it was selected based on the purposive sampling 
criteria. The criteria were health care providers who have a working experience for a minimum of 6 months in the NICU and also currently assigned at NICU.

\section{Data collection tool and method}

The tool for parental exit interview or parental satisfaction was adapted from a research done on quality of service and associated factors in NICU (16). Compliance part was prepared with consideration of Neonatal Intensive Care Unit (NICU) Training Participants' Manual 2014 and St. Paul's Hospital Millennium Medical College (SPHMMC) NICU guiding protocol February 2019 (17,38). Inventory checklist was prepared from Neonatal Intensive Care Unit (NICU) Training Participants' Manual 2014 and WHO Service Availability and Readiness Assessment (SARA) 2013 (17).

Three data collectors who are BSc midwife and BSc nurse in profession with NICU or Bemonc training background and who speak local language were recruited for parental satisfaction exit-interview and observation. Three MSc holders in midwife profession with NICU or Bemonc training background, who speak local language, were recruited for supervision.

Parental exit interview was conducted at the time of discharge from the hospital. For document and record review NICU related program documents from January 28, 2019 to July 28, 2020 GC were used and also patient cards of selected neonates were observed. Direct observations were conducted while the health care providers receive the neonate during admission to NICU ward as well as while providing services to the neonates. For key informant interview health care providers, head of the hospitals and program coordinator at zonal health office were used. Resource inventory was done in all three hospitals through both direct observation and dialogue with responsible bodies through checklist. Key informant interview, resource inventory and document review were done by the investigator himself.

\section{Data quality control}

All the data collectors and supervisors were trained by the investigator for one day about practical session on the objective of the evaluation, ethical issues while dealing with respondents, techniques of supervision and on how to use the data collections instruments.

The supervisors followed the data collection procedures closely and made immediate correction after they identified a problem. At daily bases the data collectors, supervisors and investigator were communicating at the end of the data collection time to assess the daily performance and problem encounter before conducting the next data collection and appropriate correction was given for the next day in case when problem occurs. The reliability of the satisfaction tool for all items was determined (Cronbach's alpha $=0.907$ ). Pretesting was done at Badale hospital prior to data collection period in $5 \%$ of sample size in all of the tools to check for their applicability in the local context. Parental satisfaction questionnaires was translated to Afaan Oromo or local language of the area and again cross checked with English version by independent person with health profession background.

\section{Data Management and Analysis}


The collected data were checked for completeness and consistency after the end of each data collection session. Quantitative data entry was applied using Epi-data version 3.1 and exported to SPSS version 25 statistical package for analysis. Then Descriptive analysis was done in statistical software after the data was entered to check for the presence of missing values and outliers. Parental satisfaction was rated by 10 items each having five point Likert scale from strongly disagree 1 (one) to strongly agree 5 (five). A respondent have a minimum of 10 and a maximum of 50 points on NICU service satisfaction score. Parents were categorized as dissatisfied and satisfied based a cutoff point (36.5) calculated using demarcation threshold formula ((total highest score-total lowest score)/2) + total lowest score (32). The dependent variable was dichotomized into satisfied and dissatisfied. Bivariate and multivariable logistic regression analyses were conducted to see the association between independent variable for satisfaction of parents on quality of service. Variables whose $p \leq 0.25$ were taken as candidate for multivariable logistic regression analysis and statistical significant variables were checked at $p<0.05$ after regression analysis. Adjusted odds ratio along with $95 \% \mathrm{Cl}$ was used to estimate the effect of the indicator variables on the outcome variable (20). Model fitness was checked using Hosmer and Lemeshow test and the result was 0.95 . Regarding qualitative data; qualitative data were transcribed, translated, summarized in to major thematic areas and presented in narrative form to supplement the quantitative findings. The evaluation findings were interpreted based on pre-determined judgment matrix.

\section{Matrix of Judgment and Analysis}

Matrix of judgment is the dimensions of evaluation were given weight depending on their level of relevance to the program. In each dimensions detailed indicators that used to judge the performance of NICU program were listed and assigned weight. The cumulative weight obtained from each dimension of evaluation was used to judge the program performance based on the evaluation parameters and criteria set with stakeholders based on the best information available.

\section{Results}

\section{Socio-Demographic characteristics}

A total of 109 study participants were participated on the study for parental satisfaction from the 3 primary hospitals with $100 \%$ response rate. From total study participants, $42(38.5 \%)$ were from Seka Ceqorsa primary hospital, 39 (35.8\%) were from Omo Nada primary hospital and 28 (25.7\%) were from Sexema primary hospital. From total interviewed participants 97 (89\%) were mothers of the admitted neonate and the remaining $12(11 \%)$ were fathers of the neonate. Majority $57(52.3 \%)$ of the respondents age lies between 20-29 years with an average age of 25. Most of the respondents had no formal educational background which accounts for 50 (45.9\%). From all participants, 107 (98.2\%) participants were married. From total admitted neonate during data collection period $50.5 \%$ of the neonatal age lies between 0 to 7 days during the interview time and the rest were between $8-28$ days as well as $72(66.1 \%)$ of them were male and the rest were female and their place of delivery were hospital (48.6\%), health center (39.4\%) and home (11.9\%) (Table 1). 
Table 1

Socio-demographic characteristics of study participants at the Primary Hospitals in Jimma zone, 2020 (n $=109$ ).

\begin{tabular}{|c|c|c|c|}
\hline Variables & Category & Frequency & $\begin{array}{l}\text { Percent } \\
\text { (\%) }\end{array}$ \\
\hline \multirow[t]{2}{*}{ Sex } & Male & 12 & 11 \\
\hline & Female & 97 & 89 \\
\hline \multirow[t]{2}{*}{ Relationship to admitted neonate } & Father & 12 & 11 \\
\hline & Mother & 97 & 89 \\
\hline \multirow[t]{3}{*}{ Age in years } & $<20$ years & 28 & 25.7 \\
\hline & $20-29$ years & 57 & 52.3 \\
\hline & $30-39$ years & 24 & 22 \\
\hline \multirow[t]{3}{*}{ Educational status } & No formal education & 39 & 35.8 \\
\hline & Primary level & 11 & 10.1 \\
\hline & Secondary and above & 44 & 40.4 \\
\hline \multirow[t]{2}{*}{ Marital status } & Married & 107 & 98.2 \\
\hline & Divorce & 2 & 1.8 \\
\hline \multirow[t]{2}{*}{ Occupation } & Yes & 36 & 33 \\
\hline & No & 73 & 67 \\
\hline \multirow[t]{2}{*}{ Place of residence } & Urban & 26 & 23.9 \\
\hline & Rural & 83 & 76.1 \\
\hline \multirow[t]{2}{*}{ Income } & $<750$ Ethiopian birr & 48 & 44 \\
\hline & >=750 Ethiopian birr & 61 & 56 \\
\hline \multirow[t]{2}{*}{ Age of the neonate in days } & $0-7$ & 55 & 50.5 \\
\hline & $8-28$ & 54 & 49.5 \\
\hline \multirow[t]{2}{*}{ Sex of the neonate } & Male & 72 & 66.1 \\
\hline & Female & 37 & 33.9 \\
\hline \multirow[t]{3}{*}{ Place of current child delivery } & Hospital & 53 & 48.6 \\
\hline & Health center & 43 & 39.4 \\
\hline & Home & 13 & 11.9 \\
\hline Time of hospital visit after the neonate is sick & $<12$ hours & 7 & 6.4 \\
\hline
\end{tabular}




\begin{tabular}{|llll|}
\hline Variables & Category & Frequency & $\begin{array}{l}\text { Percent } \\
\text { (\%) }\end{array}$ \\
\hline (for delivery out hospital) & Between 12-24 hours & 15 & 13.8 \\
\hline$>1$ days & 34 & 31.2 \\
\hline $\begin{array}{l}\text { Not applicable (delivery } \\
\text { at hospital) }\end{array}$ & 53 & 48.6 \\
\hline
\end{tabular}

\section{Availability of Resource}

Regarding the infrastructure of the NICU, in all 3 hospitals Kangaroo Mother Care (KMC) room, intensive care room and duty room were available. But isolation room and procedure rooms were available in only 2 of the hospitals (Seka Ceqorsa and Omo Nada). And maternal waiting area was not available in all 3 hospitals.

A 27 years old male key informant said,

"The available infrastructure is not enough to provide adequate service. When we start from the construction of the building it was not designed for NICU and it was organized here from available rooms in collaboration with hospital administration."

Again he said, "... As we know neonates who are admitted in this ward needs intensive care and parents of the neonate can visit and spend time with their babies who stay in the NICU for a few times. But there is no area for mothers of newborn to wait in the unit until they contact with their babies. Due to this we don't know where the parents wait whether the area is clean or safe. Then they came back and contact with their neonate which may expose the neonate to infections."

Regarding the laboratory investigations, essential medical supplies and equipment's, in all hospitals drugs like Ampicillin IV, Gentamycin IV, Ceftriaxone IV, Metronidazole IV, TTC, Phenobarbitone, Glucose were available and equipment's like suction machine, pulse oximeter, Ambubag, radiant warmer, thermometer, weight scale for babies, stethoscope, gloves were also available in all hospitals. Drugs i.e. cloxacillin, chlorhexidine, vitamin $\mathrm{k}$ were not available in Omo Nada hospital and acyclovir was not available in all hospitals. Medical equipment's like continuous positive airway pressure (CPAP) was not available in all hospitals. Laboratory service were available in all hospitals including investigations like $\mathrm{CBC}$, urine analysis, RBS, stool examination and chest x-ray were available in hospitals but provided in collaboration with other services.

A 30 years old male key informant said,

"... Since we started this department despite we do have a lot of resources shortages. And we have tried to mobilize resources from the near back hospitals such as incubators, phototherapy machines and etc. 
There is no single equipment or instrument that is supplied by the Oromia Regional Health Department to start this NICU service."

A 25 years old male key informant said,

"In this hospital neonates are referred to higher level mainly with two reasons the first one is due to lack of bilirubin testing in the laboratory so we refer patients with neonatal jaundice and the second is lack of CPAP machine."

Concerning human resource in all primary hospitals, a total of 13 nurses were available with an average of 4 nurses per each hospital in the NICU. From the 12 nurses, 3 were trained from Seka Ceqorsa, 2 were trained from Sexema and 1 was trained from Omo Nada hospital about NICU services. Laboratory and pharmacists were working in collaboration with other activities in all hospitals. Cleaners were working in collaboration with other wards except Seka Cekorsa hospital were 1 cleaner assigned only for NICU.

A 25 years old male key informant said,

"... all staffs are not trained on NICU. If the training is provided to all staffs working in the NICU, all of us will have the same idea and perform our job effectively. There are a number of equipment in this intensive care room that needs adjustments for example we were referring neonates who are in need of incubator due to lack of knowledge on its adjustment. But currently, one of our staff already received the training and adjusted the incubator which helped us to reduce the referral due to incubator."

A 33 years old male key informant also said,

"...the NICU training is given for health workers for at least one month which needs high resources and its above our capacity. So, the training is mainly provided at regional level. However we conduct review meeting in collaboration with other programs."

On the inventory of recording and reporting format there were low achievements with an average availability of $42.2 \%$. Discharge criteria, follow-up schedule and normal value charts were not documented or posted in the wall of the room in all hospitals however registrations and reporting formats were available in all hospitals.

Overall, $88.32 \%$ of the required resources were available with the judgment category of successfully implemented according to the pre-set criteria (Table 2). 
Table 2

Analysis and Judgment Matrix for availability dimension of the evaluation of quality of NICU at Jimma zone primary Hospitals, 2020.

\begin{tabular}{|c|c|c|c|c|c|c|}
\hline $\begin{array}{l}\text { S. } \\
\text { No }\end{array}$ & Indicators & $\begin{array}{l}\text { Expected } \\
\text { in } \\
\text { number }\end{array}$ & $\begin{array}{l}\text { Observed } \\
\text { in } \\
\text { number }\end{array}$ & $\begin{array}{l}\text { Weight } \\
\text { (W) }\end{array}$ & $\begin{array}{l}\text { Score } \\
\text { (S) }\end{array}$ & $\begin{array}{l}\text { Achievement } \\
\text { in \% } \\
(\mathrm{S} / \mathrm{W} 100)\end{array}$ \\
\hline 1 & $\begin{array}{l}\text { Number of hospitals with at least } \\
\text { two trained health care provider in } \\
\text { NICU }\end{array}$ & 6 & 9 & 5 & 5 & 100 \\
\hline 2 & $\begin{array}{l}\text { Number of hospital's with } \\
\text { neonatal bed in the NICU ward }\end{array}$ & 3 & 3 & 5 & 5 & 100 \\
\hline 3 & $\begin{array}{l}\text { Number of hospitals with } \\
\text { adequate NICU rooms }\end{array}$ & 3 & 3 & 4 & 4 & 100 \\
\hline 4 & $\begin{array}{l}\text { Number of hospitals with medical } \\
\text { equipment's needed to provide } \\
\text { NICU service }\end{array}$ & 3 & 3 & 3 & 3 & 100 \\
\hline 5 & $\begin{array}{l}\text { Number of hospitals with drug } \\
\text { supplies }\end{array}$ & 3 & 3 & 5 & 5 & 100 \\
\hline 6 & $\begin{array}{l}\text { Number of hospitals with } \\
\text { functional laboratory service }\end{array}$ & 3 & 3 & 6 & 6 & 100 \\
\hline 7 & $\begin{array}{l}\text { Number of hospitals with NICU } \\
\text { guideline placed in NICU room }\end{array}$ & 3 & 3 & 4 & 4 & 100 \\
\hline 8 & $\begin{array}{l}\text { Number of hospitals with one } \\
\text { standard NICU registration book }\end{array}$ & 3 & 3 & 6 & 6 & 100 \\
\hline 9 & $\begin{array}{l}\text { Number of hospitals with } \\
\text { standard monthly reporting } \\
\text { format }\end{array}$ & 3 & 3 & 5 & 5 & 100 \\
\hline 10 & $\begin{array}{l}\text { Number of hospitals with tap } \\
\text { water in the NICU ward }\end{array}$ & 3 & 0 & 4 & 0 & 0 \\
\hline 11 & $\begin{array}{l}\text { Number of hospitals with } \\
\text { functional electricity in NICU }\end{array}$ & 3 & 3 & 6 & 6 & 100 \\
\hline Tot & & & & & & $88.32 \%$ \\
\hline
\end{tabular}

\section{Compliance to Guideline}

The compliance dimension was assessed using direct observation of patient-provider interaction and document review in all the 3 hospitals. A total of 15 patient-provider interaction (5 per each hospital) session were observed in all 3 hospitals. The result showed that in all of the sessions observed the provider putted the neonate on sterile sheet but in only $66.67 \%$ of the observations the provider greet the 
parents of the neonate on admission. In addition, all providers in the hospitals measure weight, temperature, respiratory rate and apical heartbeat of the neonate as well as counseling was given to the families of the admitted neonate in their language that they easily can understand.

In all hospitals there were national standard NICU register. Six month records (from June 27, 2019 to December 30, $2019 \mathrm{EC}$ ) of NICU register were done to assess the compliance in all 3 hospitals. There were 335 total recorded cases in all 3 hospitals (131 cases in Seka Cekorsa, 85 cases in Sexema and 119 cases in $\mathrm{Omo} \mathrm{Nada)} \mathrm{in} \mathrm{the} \mathrm{register.} \mathrm{The} \mathrm{most} \mathrm{common} \mathrm{causes} \mathrm{of} \mathrm{admission} \mathrm{were} \mathrm{neonatal} \mathrm{sepsis}$ accounts for $114(34.03 \%)$ followed by prematurity 46 (13.73\%) and Respiratory Distress Syndrome (RDS) 32 (9.55\%) from all recorded cases (Fig. 1).

In all of the recorded cases personal identification, admission problem and management of the neonate part were recorded in the register. However, admission information, delivery information, maternal condition and discharge information part were recorded only in $226(67.5 \%)$ of the recorded cases in the register. On the other hand, supportive supervision from zonal health office to hospitals was undertaken once in one hospital only in two quarters. This is supported by qualitative finding,

"... and as I remember there is no any regular supportive supervision to the NICU from the higher officials or higher department from zonal or from regional level. But we do have the internal supervision here in order to tackle those problems."

From total observed patient cards only $26.67 \%$ of them were appointed for follow -up within the first week of after they were discharged from the hospital. This was supported by the finding in the qualitative data.

A 25 years old male key informant said,

"... For example neonates who are managed with prematurity, asphyxia need follow-up but we are not providing to them until now. Currently, we are discussing with staffs to start follow-up."

Overall, the compliance of health care providers to the guideline were $82.59 \%$ with the judgment category of partially implemented according to the preset criteria (table 3 ). 
Table 3

Analysis and Judgment matrix for compliance of the evaluation of quality of NICU at Jimma zone primary Hospitals 2020

\begin{tabular}{|c|c|c|c|c|c|c|}
\hline S.N. & Indicators & $\begin{array}{l}\text { Expected } \\
\text { in } \\
\text { number }\end{array}$ & $\begin{array}{l}\text { Observed } \\
\text { in } \\
\text { number }\end{array}$ & $\begin{array}{l}\text { Weight } \\
\text { (W) }\end{array}$ & $\begin{array}{l}\text { Score } \\
\text { (S) }\end{array}$ & $\begin{array}{l}\text { Achievement } \\
\text { in \% } \\
(\mathrm{S} / \mathrm{W}+100)\end{array}$ \\
\hline 1 & $\begin{array}{l}\text { Proportion of neonates who are } \\
\text { putted on sterile sheet on } \\
\text { admission }\end{array}$ & 15 & 15 & 12 & 12 & 100 \\
\hline 2 & $\begin{array}{l}\text { Proportion of neonates who are } \\
\text { weighted on admission }\end{array}$ & 15 & 15 & 15 & 15 & 100 \\
\hline 3 & $\begin{array}{l}\text { Proportion of neonates who are } \\
\text { measured temperature on } \\
\text { admission }\end{array}$ & 15 & 15 & 15 & 15 & 100 \\
\hline 4 & $\begin{array}{l}\text { Proportion of HW provide } \\
\text { counselling to parents during } \\
\text { admission }\end{array}$ & 15 & 15 & 9 & 9 & 100 \\
\hline 5 & $\begin{array}{l}\text { proportion of neonates who are } \\
\text { continuously monitored during } \\
\text { admission }\end{array}$ & 15 & 15 & 10 & 10 & 100 \\
\hline 6 & $\begin{array}{l}\text { Proportion of neonates who are } \\
\text { appointed within } 7 \text { days of after } \\
\text { discharge }\end{array}$ & 15 & 4 & 9 & 2.4 & 26.67 \\
\hline 7 & $\begin{array}{l}\text { Proportion of hospitals which } \\
\text { received quarterly integrated SS } \\
\text { for the last } 6 \text { months }\end{array}$ & 6 & 1 & 9 & 1.5 & 16.67 \\
\hline 8 & $\begin{array}{l}\text { \# of timely report compiled for } \\
\text { the last } 2 \text { quarter }\end{array}$ & 18 & 18 & 10 & 10 & 100 \\
\hline 9 & $\begin{array}{l}\text { \# of complete report compiled } \\
\text { for the last } 2 \text { quarter }\end{array}$ & 18 & 18 & 11 & 11 & 100 \\
\hline Total & & & & & & 82.59 \\
\hline
\end{tabular}

\section{Satisfaction of Parents with the NICU services}

A total of 109 participants were participated on the study. Majority of the respondents, 75 (68.8\%), were satisfied with cleanness of the NICU. From all participants 89 (81.6\%) of them were satisfied with the availability of service provider on working time. From total respondents $85(77.9 \%)$ were satisfied with the staffs response about any information. Regardless of the availability of neonatal bed in the NICU, 60 (55.1\%) of the participants were satisfied. Almost 86 (78.9\%) of the participants were satisfied on their babies progress. Out of ordered laboratory investigation 87 (79.8\%) of the participants were satisfied with availability of all ordered laboratory investigations. 
Majority 76 (69.8\%) of the participants were satisfied with the availability of all prescribed drugs for their neonate. Nearly 93 (85.4\%) of the study participants were satisfied with supports of the staff and it was the highest rate by participants on this study. About 56 (51.4\%) were satisfied with the room ventilation. From total participants 86 (78.9) of them responded that they satisfied with the adequate treatment provided to their admitted neonate. Overall, the proportion of parents who satisfied with services given in the NICU ward was $72.75 \%$ in 5-Likert scale with the judgment category of partially implemented according to the preset criteria (Table 4). 
Table 4

Analysis and Judgment matrix for satisfaction of study participants in Jimma zone primary Hospitals, 2020.

\begin{tabular}{|c|c|c|c|c|c|c|}
\hline S.N. & Indicators & $\begin{array}{l}\text { Expected } \\
\text { in } \\
\text { number }\end{array}$ & $\begin{array}{l}\text { Observed } \\
\text { in } \\
\text { number }\end{array}$ & $\begin{array}{l}\text { Weight } \\
\text { (W) }\end{array}$ & $\begin{array}{l}\text { Score } \\
\text { (S) }\end{array}$ & $\begin{array}{l}\text { Achievement } \\
\text { in \% } \\
\left(\mathrm{S} / \mathrm{W}^{*} 100\right)\end{array}$ \\
\hline 1 & $\begin{array}{l}\text { Proportion of Parents who } \\
\text { satisfied with cleanliness of } \\
\text { NICU }\end{array}$ & 109 & 75 & 10 & 6.88 & 68.8 \\
\hline 2 & $\begin{array}{l}\text { Proportion of Parents who } \\
\text { satisfied with the availability of } \\
\text { service provider on working time }\end{array}$ & 109 & 89 & 10 & 8.17 & 81.7 \\
\hline 3 & $\begin{array}{l}\text { Proportion of Parents who } \\
\text { satisfied with the staffs } \\
\text { response about any information }\end{array}$ & 109 & 85 & 10 & 7.8 & 78 \\
\hline 4 & $\begin{array}{l}\text { proportion of Parents who } \\
\text { satisfied with availability of } \\
\text { neonatal bed in NICU }\end{array}$ & 109 & 60 & 10 & 5.5 & 55 \\
\hline 5 & $\begin{array}{l}\text { Proportion of Parents who } \\
\text { satisfied with their baby's } \\
\text { progress }\end{array}$ & 109 & 86 & 10 & 7.89 & 78.9 \\
\hline 6 & $\begin{array}{l}\text { Proportion of Parents who } \\
\text { satisfied with availability of all } \\
\text { ordered investigation }\end{array}$ & 109 & 87 & 10 & 7.98 & 79.8 \\
\hline 7 & $\begin{array}{l}\text { Proportion of Parents who } \\
\text { satisfied with availability of all } \\
\text { prescribed drug }\end{array}$ & 109 & 76 & 10 & 6.97 & 69.7 \\
\hline 8 & $\begin{array}{l}\text { Proportion of Parents who } \\
\text { satisfied with supports of the } \\
\text { staff }\end{array}$ & 109 & 93 & 10 & 8.53 & 85.3 \\
\hline 9 & $\begin{array}{l}\text { Proportion of Parents satisfied } \\
\text { with room's ventilation }\end{array}$ & 109 & 56 & 10 & 5.14 & 51.4 \\
\hline 10 & $\begin{array}{l}\text { Proportion of Parents satisfied } \\
\text { with adequate treatment for } \\
\text { neonate }\end{array}$ & 109 & 86 & 10 & 7.89 & 78.9 \\
\hline Total & & & & & & 72.75 \\
\hline
\end{tabular}

\section{Overall quality of NICU services}

In summary when we see the overall quality of NICU in Jimma zone primary hospitals it was $82.42 \%$ in 3 dimensions (Availability $=88.32 \%$, Compliance $=82.59 \%$ and Satisfaction $=72.75 \%$ ) with the category of partially implemented based on the preset judgment matrix (Table 5). 
Table 5

Overall judgment matrix and analysis of the evaluation of quality of NICU at Jimma zone primary hospitals, 2020.

\begin{tabular}{|c|c|c|c|c|c|}
\hline S.N. & Dimension & $\begin{array}{l}\text { Present } \\
\text { achievement }\end{array}$ & $\begin{array}{l}\text { Agreed } \\
\text { weight }\end{array}$ & $\begin{array}{l}\text { Value } \\
\text { Achieved }\end{array}$ & Judgment criteria \\
\hline 1 & Availability & 88.32 & 40 & 35.33 & $\begin{array}{l}>85 \%=\text { successfully } \\
\text { implemented }\end{array}$ \\
\hline 2 & Compliance & 59 & 35 & 28.91 & $\begin{array}{l}70 \%-85 \%=\text { partially } \\
\text { implemented }\end{array}$ \\
\hline 3 & Satisfaction & 72.75 & 25 & 18.18 & $55-69.9 \%=$ fairly implemented \\
\hline \multicolumn{3}{|c|}{ Total Score } & 100 & 82.42 & $\begin{array}{l}\leq 54.9 \%=\text { not successfully } \\
\text { implemented }\end{array}$ \\
\hline
\end{tabular}

\section{Factors associated with Parents satisfaction with NICU services}

Eight variables such as sex, age, income, educational status, marital status, and occupation, place of residence and place of delivery were checked in the bivariate model for any significant association with parental satisfaction. Out of these variables three variables such as educational status, occupation and place of residence had shown association in bivariate logistic regression and they were candidate for multivariable logistic regressions at $\mathrm{p}$-value less than 0.25 .

In the multivariable logistic regression analysis we have identified place of residence and occupation as predictors for satisfaction of parents on the NICU services. According to the findings, parents who came from rural were 3.68 times more likely to be satisfied on neonatal intensive care services than those from urban (AOR $=3.68,95 \% \mathrm{Cl}: 1.15-11.76)$. And parents who had occupation were 2.69 less satisfied than those who have occupation on NICU service (AOR = 2.69, 95\% Cl: 1.01-7.19) (Table 6). 
Table 6

Multivariable logistic regression analysis result of overall parental satisfaction in the evaluation of quality of NICU at Jimma zone primary hospitals, $2020(n=109)$

\begin{tabular}{|c|c|c|c|c|c|}
\hline \multicolumn{2}{|l|}{ Variable } & \multicolumn{2}{|c|}{ Parents satisfaction } & \multirow{2}{*}{$\begin{array}{l}\text { Crude OR with } \\
95 \% \mathrm{Cl}\end{array}$} & \multirow{2}{*}{$\begin{array}{l}\text { Adjusted OR with } \\
95 \% \mathrm{Cl}\end{array}$} \\
\hline Name & Category & $\begin{array}{l}\text { Dissatisfied } \\
(\%)\end{array}$ & $\begin{array}{l}\text { Satisfied } \\
(\%)\end{array}$ & & \\
\hline \multirow[t]{2}{*}{$\begin{array}{l}\text { Place of } \\
\text { residence }\end{array}$} & Urban & $15(57.7)$ & $\begin{array}{l}11 \\
(42.3)\end{array}$ & 1 & 1 \\
\hline & Rural & $17(20.5)$ & $\begin{array}{l}66 \\
(79.5)\end{array}$ & $\begin{array}{l}5.29(2.06,13.59) \\
*\end{array}$ & $3.68(1.15,11.76)^{x}$ \\
\hline \multirow[t]{3}{*}{$\begin{array}{l}\text { Educational } \\
\text { status }\end{array}$} & $\begin{array}{l}\text { No formal } \\
\text { education }\end{array}$ & $13(26)$ & 37 (74) & 1 & 1 \\
\hline & Primary level & $10(22.7)$ & $\begin{array}{l}34 \\
(77.3)\end{array}$ & $1.74(1.22,3.45)$ & $1.65(.57,4.72)$ \\
\hline & $\begin{array}{l}\text { Secondary level } \\
\text { and above }\end{array}$ & $9(60)$ & $6(40)$ & $.23(.07,0.78)^{\star}$ & $.95(.21,4.36)$ \\
\hline \multirow[t]{2}{*}{ Occupation } & Yes & $18(50)$ & $18(50)$ & 1 & 1 \\
\hline & No & $14(19.2)$ & $\begin{array}{l}59 \\
(80.8)\end{array}$ & $\begin{array}{l}4.21(1.75 \\
10.11)^{\star}\end{array}$ & $\begin{array}{l}2.69(1.01 \\
7.191)^{\star}\end{array}$ \\
\hline
\end{tabular}

\section{Discussion}

This study was conducted to evaluate the quality of neonatal intensive care unit in Jimma zone primary hospitals in terms of availability of materials, compliance of health care workers towards the NICU guideline and parental satisfaction related to overall service provided to their neonates in the unit.

Accordingly, most of the relevant resources being used for NICU service were available in primary hospitals of Jimma Zone. However, some key resources such as continuous positive airway pressure, laboratory reagent, service provision rooms, water supply, communication phone, infection prevention guideline and patient follow-up card, were scarce in the hospital units. Hence, these constraints cause difficulties in reaching both national and international standards.

For instance, the Ethiopian NICU guideline states the availability of skilled health providers; reliable electricity and water supplies are important aspects in providing quality services in the hospitals (9). In addition, the American NICU Consensus Committee suggested that the hand washing station should be hands-free hand washing station with no hand contact when opening and closing the water and should be placed within 1 to 6 meters of each infant bed (21). However, the set up in Jimma Zone primary hospitals does not meet both national and international standards. So that, our finding tells us in all 3 
hospital tap water in the ward was not available which increases the risk of contamination for the patients and providers.

Human resources are the other important resources at NICU. The national manual recommendation requires provision of NICU service with skilled providers. In line with this, there was one pediatrician nurse, 3 general practitioners and 13 nurses at the NICU in all three hospitals. A study at Debre Birhan Referral Hospital showed that one pediatrician, two general practitioners and 15 nurses were available at NICU (16). This difference might be associated with the level of hospitals, where our study includes primary hospitals.

In addition, in terms of the structure of NICU this study finds in all three hospitals essential drug supplies and medical equipment's such as Radiant warmer, suction machine, pulse oximeter were available in the neonatal intensive care unit. However, equipment's such as Continuous Positive Airway Pressure (CPAP) were not available in all 3 hospitals. This finding was contrary to the national guideline which states that CPAP should be initiated for preterm newborns with Respiratory Distress Syndrome (9). Similarly, a study done on Debre Birhan Referral Hospital on the quality of service and associated factors in NICU shows that medical equipment's such as pulse oximetry and CPAP were not available (16). This difference on the availability of pulse-oximetry on the two studies may be due to an increased government concern towards the program.

In this study, we find that in all hospitals there is a shortage of laboratory reagent for performing bilirubin tests and managing neonates with jaundice after diagnosis. As a result neonates with this condition were referred to Jimma hospital for diagnosis and management. This finding was consistent with a rapid assessment conducted on the quality of neonatal health care in Kilimanjaro region northeast Tanzania that revealed none of the facilities had capacity to perform bilirubin test (22).

NICU training manual recommended that, when managing a neonate with a problem in the NICU to use the normal value charts. It is critically needed that sticking to the figures and the recommended values to closely follow the prognosis of the sick neonate and avoid unwanted outcomes of the treatment and management applied on the newborn under care. For quick reference the guideline advised that to print the normal value charts and posted them on the visible area on the wall in the NICU (23). However, the findings in all hospital contradict with the NICU protocol in that normal value charts were not posted on the wall of NICU in all hospitals.

According to St. Paul's Hospital Millennium Medical College NICU protocol 2019, during the admission of patients all babies have to be received on sterile sheet as well as weight and temperature of the baby should have to be recorded during admission. These activities used to prevent the babies from hospital acquired infections. Regarding this, in all hospitals health care providers were receiving the neonate on sterile sheet, examining weight and temperature on admission (19).

In this study, only $26.67 \%$ of admitted neonates received follow-up after being discharged. Follow-up is needed to provide continuum of specialized medical management for neonates admitted, treated, and 
discharged from NICU. This finding is contradictory to the National NICU guideline which indicated that all neonates has to be appointed within the first 7 days following discharge from hospital to see if the child is adopting the home environment (23).

Follow-up while on admission is critical that sick neonates are closely followed up 24 hours a day, seven days a week and throughout their stay in the health facilities. Based on the specific diagnosis they have and their general conditions, the level of follow up they may need may vary. And the follow-up must be recorded on chart that is used for the daily and hourly monitoring of the sick neonates to ensure that the progress of the problem and the condition of the sick neonate at each point (23). So follow-up of vital signs for the admitted babies were an important activity on the prevention of complications and for monitoring babies progress. During observation in the sampled hospitals health care providers were providing continuous monitoring for the admitted neonates in all hospitals.

In neonatology, the patient is the baby, but the parent is the recipient of information. Many parents want as much information as possible about the diseases that affect their children. Unfortunately, the NICU is a complex clinical setting where information and predictions vary and change rapidly: decisions are complex, and parents find it difficult to cope with the uncertainty. It is a good practice for parents to be present at the bedside during NICU rounds. This helps them to be updated regularly and to become familiar with all team members. The study found that health care providers provide counseling in their local language to the neonate's parents, so that parents can understand it easily (23).

Supportive supervision is a facilitative approach which promotes mentoring, joint problem-solving and communication between supervisors and hospitals and used to improve the routine program monitoring and evaluation activities. In line with this, the national NICU guideline also states that routine supportive supervision of the health facilities with NICU also used to monitor NICU service and program data (23). However this study revealed that, only one hospital received supportive supervision from the zonal health office once within 6 months, and had a document of that.

Given that paternal satisfaction with neonatal services is an important measure of quality of care and provision of services, this evaluation discovered the parental satisfaction towards the services given to their neonate. Accordingly, the overall satisfaction of parents satisfied with the NICU services was about $72.75 \%$. This study finding showed better parental satisfaction than a study conducted at Tikur Anbessa Specialized Hospital which is $65 \%$ overall satisfaction and lower than a study conducted at Debre Birhan Referral Hospital which is $77 \%(16,20)$. This difference may be due to the difference in literacy of respondents and satisfaction items.

In this study, place of residence and occupation were significantly associated with parental satisfaction with NICU services after controlling for other independent variables. This means that parents from urban areas had lower perceived satisfaction than rural residents and parents with occupation had also low perceived satisfaction on the quality of care in NICU as compared to without occupation. This may seem obviously related to the fact that urban residents and parents with occupation would have higher expectation when visiting a health facility. This study finding is supported by the study conducted in 
Gondar University Specialized teaching Hospital on mothers satisfaction where place of residence is a strong predictor of satisfaction.

\section{Limitation of study}

Health care provider might perform in a more appropriate and acceptable way and they may want to make things seem better than they are during direct observation of patient-provider interaction (Hawthorn effect). To prevent this, the first two observations were discarded in each hospital and the trained data collectors maintained a distance from the observed area.

\section{Conclusion}

This evaluation result concluded that the overall quality of neonatal intensive care unit service in Jimma zone primary hospitals was implemented partially per the preset judgment criteria agreed with key stakeholders during evaluability assessment phase. Availability of resources in neonatal intensive care unit in Jimma zone primary hospitals were in acceptable range. However, it requires improvements in fulfilling additional materials. The compliance dimension of evaluation fall under partially implemented category according to the judgment matrix. Parental satisfaction was considered as partially implemented category in preset judgment criteria. The predictors of parental satisfaction in the services given in the NICU were place of residence and occupation.

Health professionals have to provide continuous follow-up to neonates discharged from the NICU according to the national guideline s567843chedule. Posting or documenting normal value charts, discharge criteria, isolation criteria and follow-up schedule in the place where every staff can access it easily. Hospitals should prepare hand washing facilities in the NICU ward and prepare patient follow-up card for discharged neonates. Regular supportive supervision and training should have to be in place to support and monitor hospitals.

\section{Abbreviations}

AOR: Adjusted odds ratio; Cl: Confidence interval; COR: Crude odds ratio; CPAP: Continuous positive airway pressure; NICU: Neonatal intensive care unit; WHO: World health organization.

\section{Declarations}

\section{Ethics approval and consent to participate}

Permission for data access was obtained from Jimma University, College of Health and Medical Sciences Institutional Health Research Ethics Review Committee (IHRERC). After clear discussion about the actual study or explaining of purpose of the study and the confidentiality issues, informed consent was obtained from the study participants. Identification of study participants by name was avoided to assure the 
confidentiality of the information obtained. Data collectors assured that the data was handled confidentially. All methods were performed in accordance with the relevant guidelines and regulations.

\section{Consent for publication}

Not applicable for this study.

\section{Competing interests}

The authors declare that they have no competing interests.

\section{Acknowledgment}

At the very beginning thank to the almighty GOD for all aspects of my life. Next to this my deepest admiration, gratitude and thanks go to Jimma University for their facilitation and allowing me to carry out this evaluation thesis.

Last but not the least I would like to thank Jimma zone Health office, all the primary hospitals and head of the hospitals for accepting and encouraging to do the evaluation and dedicating their time during evaluability assessment and providing the necessary information which I need for the evaluation of the program.

\section{Contribution of Authors}

Proposal preparation, acquisition of data, analysis, and interpretation of data was done by L.D., K.A. and, Y.H instructs the study design data cleaning and analysis. YH drafted the manuscript and all authors have a substantial contribution in revising and finalizing the manuscript. All authors read and approved the final manuscript.

\section{Funding}

This research received no specific grant from any funding agency in the public, commercial or no forprofit sectors.

\section{Disclosure}

The authors received no financial support to be disclosed 


\section{Patient and public involvement}

Patients and/or the public were not involved in the design, or conduct, or reporting, or dissemination plans of this research.

\section{Patient consent for publication}

Not required.

\section{Data availability statement}

All documentary data and literature relevant to the study are publicly available. Interview data will not be shared to maintain confidentiality.

\section{References}

1. UNICEF, WHO, World Bank Group, United Nations. Levels and Trends in Child Mortality: Estimates Developed by the UN Inter-agency Group for Child Mortality Estimation. 2019;52.

2. Opportunities for Africa's Newborns.

3. Institute of public health, ICF. Mini Demographic and Health Survey: key indictors [Internet]. Handbook of Federal Countries, 2005. 2019. 136-148. Available from: www.DHSprogram.com.

4. Newborn N, Strategy CS, Summary DB. 2015/16-2019/20. 2019; (June 2015).

5. Fata GA. Causes and factors associated with neonatal mortality in Neonatal Intensive Care Unit (NICU) of Jimma University Medical Center, Jimma , South. 2019;39-48.

6. Orsido TT, Asseffa NA, Berheto TM. Predictors of Neonatal mortality in Neonatal intensive care unit at referral Hospital in Southern Ethiopia: A retrospective cohort study. BMC Pregnancy and Childbirth. 2019; 19 (1):1-9.

7. Federal Democratic Republic of Ethiopia Ministry of Health. May 2015. 2019;20 (May 2015).

8. FMOH, WHO U. Integrated Management of Newborn and Childhood Illness, Part 1. Module. 2012;1(1).

9. Neonatal Intensive Care Unit (NICU) Implementation Guide (Draft). 2016;

10. Intensive Care Society; Core Standards for Intensive Care Units. 2013;1:1-27.

11. Product K. Developing newborn intensive care units; Knowledge Product. 2018 ;(June). Available from: www.vsointernational.org

12. National Strategy for Child Survival in Ethiopia. 2015 ;(June).

13. Worku B, Kidane L, Abera K, Kumar A, Egeli P KY. Improving Neonatal Health outcomes in Ethiopia through an innovative and sustainable Health care model; [Internet]. 2016;XIII, No.2. Available from: http://ejol.aau.edu.et/index.php/EJPC/article/download/677/644/ 
14. CSA. Summary and statistical report of the 2007 population and housing census. Addis Ababa, Ethiopia. Federal democratic republic of Ethiopia population census commission. 2008;1-1084.

15. Donabedian A. An introduction to quality assurance in Health Care; Oxford University Press. Inc.; 2003.

16. Wassie Negash Mekonnen AMD; Quality of Service and Associated Factors in Neonatal Intensive Care Unit at Debre Berhan Referral Hospital, Debre Berhan Ethiopia: A Cross sectional Study. 11(5):1-8.

17. Ababa A. Neonatal Intensive Care Unit (NICU) Training Participants' Manual. 2014 ;( November).

18. O'Neill K, Sheffel A. Service Availability and Readiness Assessment (SARA) An annual monitoring system for service delivery Reference Manual. 2013;1-168. Available from http://www.who.int/about/licensing/copyright_form/en/index.html

19. Protocol G. Sphmmc nicu. 2019;

20. Kibret Gt, Radie Yt. Parental Satisfaction And Involvement Concerning Care Of Their Hospitalized Child. 2019;7(3):341-62.

21. White RD, Smith JA, Shepley MM. Recommended standards for newborn ICU design, eighth edition. Journal of Perinatology. 2013; 33(SUPPL. 1):2-16.

22. Mbwele B, Reddy E, Reyburn H. A rapid assessment of the quality of neonatal healthcare in Kilimanjaro region, northeast Tanzania. BMC Pediatrics. 2012;12(November 2012).

23. Protocol M. Neonatal Intensive Care Unit ( NICU ) Training. 2014;(March).

\section{Figures}




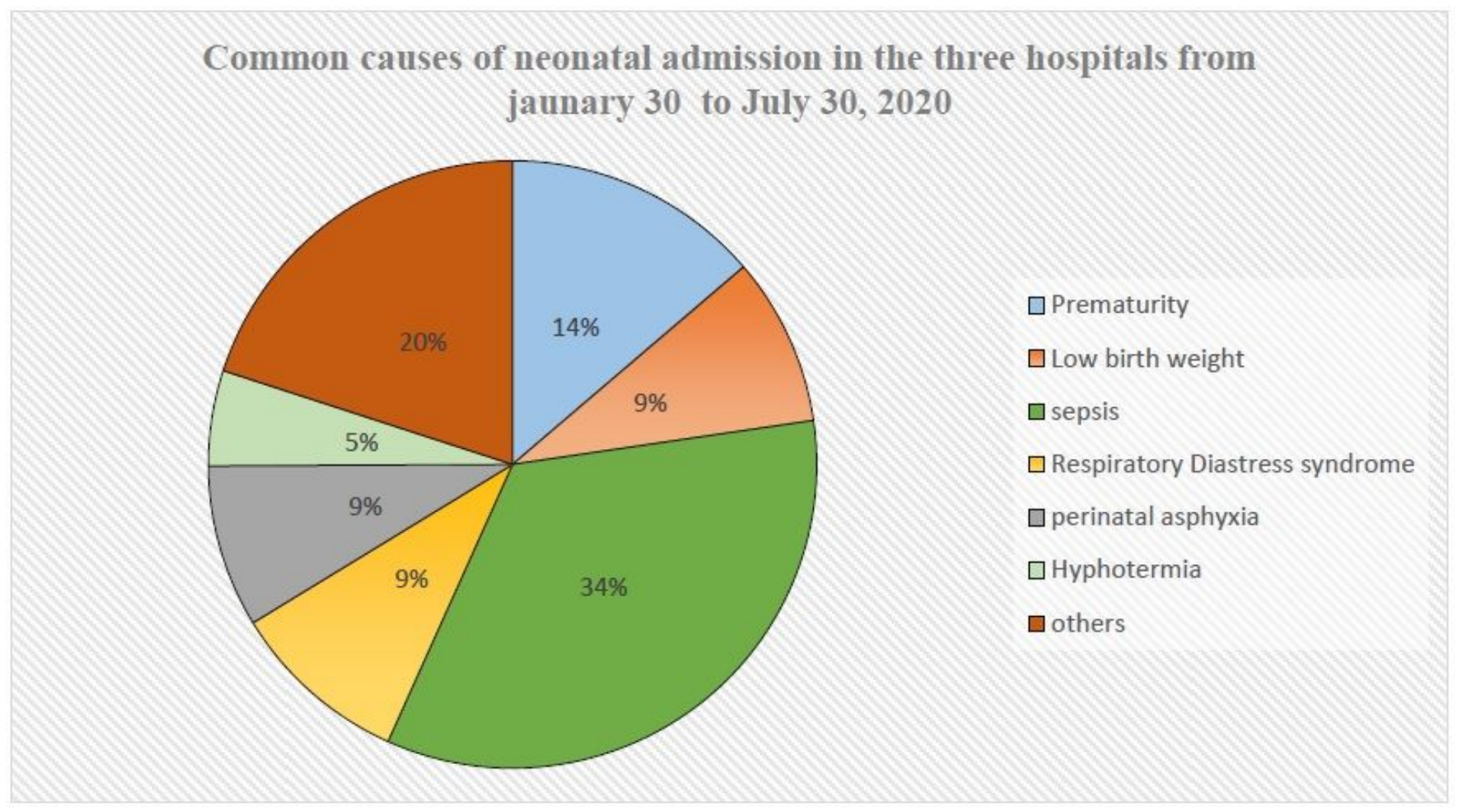

\section{Figure 1}

common causes of neonatal admission in Jimma zone primary hospital with NICU service from Jaunary 30 to July 30,2020 . 